\title{
Nematodes can survive in a suspended form of life for indefinite time
}

Anastasia Shatilovich ${ }^{1 \#}$, Vamshidhar R. Gade ${ }^{2,9 \#}$, Martin Pippel ${ }^{3}$, Tarja T. Hoffmeyer ${ }^{4}$, Alexei V. Tchesunov ${ }^{5}$, Lewis Stevens ${ }^{6}$, Sylke Winkler ${ }^{2,7}$, Graham M. Hughes ${ }^{8}$, Sofia Traikov ${ }^{2}$, Michael Hiller ${ }^{3,10}$, Elizaveta Rivkina ${ }^{1}$, Philipp H. Schiffer ${ }^{4 *}$, Eugene W Myers ${ }^{3}$, Teymuras V. Kurzchalia ${ }^{2 *}$

1. Institute of Physicochemical and Biological Problems in Soil Science RAS, Pushchino, Russia

2. Max Planck Institute for Molecular Cell Biology and Genetics, Dresden, Germany

3. Center for Systems Biology, Dresden, Germany

4. Institute for Zoology, University of Cologne

5. Department of Invertebrate Zoology, Faculty of Biology, M.V. Lomonosov Moscow State University, Moscow, Russia

6. Tree of Life, Wellcome Sanger Institute, Cambridge, UK

7. DRESDEN concept Genome Center, Dresden, Germany

8. School of Biology and Environmental Science, University College Dublin, Belfield, Dublin, Ireland

9. Institute of Biochemistry, ETH Zürich, 8093 Zürich, Switzerland

10. LOEWE Centre for Translational Biodiversity Genomics, Senckenberg Society for Nature Research \& Goethe University, Frankfurt am Main, Germany

\footnotetext{
\# A.S and V.G contributed equally to this work

*To whom correspondence may be addressed

Email: kurzchalia@mpi-cbg.de

p.schiffer@,uni-koeln.de
} 


\begin{abstract}
When environmental conditions are unfavorable, such as the complete absence of water or oxygen, high temperature, freezing or extreme salinity, some organisms can enter suspended animation (cryptobiosis) ${ }^{1}$. This reversible transition is preceded by execution of complex genetic and biochemical programs (preconditioning) ${ }^{2,3,4}$. Under laboratory conditions, however, animals have only been maintained in a viable cryptobiotic state for a short time. Here we show that desiccation followed by freezing allows C. elegans dauer larvae to retain full viability over very long periods (around 500 days). Consistent with this finding, recently nematode individuals have been reanimated from the Siberian permafrost ${ }^{5}$, that according to precise radiocarbon dating shows that they remained in cryptobiosis since the late Pleistocene, for about 46,000 years. Phylogenomic inference based on our high-quality genome assembly and morphological analysis demonstrate that these nematodes belong to a novel parthenogenetic species, which we named Panagrolaimus kolymaensis. Genome analysis revealed that the core of the molecular toolkit for cryptobiosis in P. kolymaensis and C. elegans is orthologous. To survive desiccation and freezing under laboratory conditions these two species display similar biochemical responses. Thus, nematodes possess extraordinarily robust adaptive mechanisms that potentially allow them to remain in suspended animation over geological time scales.
\end{abstract}




\section{Main}

Organisms from various taxonomic groups can survive extreme and otherwise lethal conditions such as the complete absence of water or oxygen, high temperature, freezing or extreme salinity. The survival strategies of such organisms include a state known as suspended animation, or cryptobiosis, in which they reduce their metabolism to an undetectable level ${ }^{1}$. Spectacular examples of long-term cryptobiosis include a Bacillus spore that was preserved in the abdomen of bees buried in amber for 25 to 40 million years ${ }^{6}$, and a 1000 to 1500 years-old Lotus seed found in an ancient lake that was subsequently able to germinate ${ }^{7}$. Metazoans such as tardigrades, rotifers and nematodes are also known for remaining in cryptobiosis for prolonged periods $^{8,9}$. In 2018, we discovered that nematodes from the Siberian permafrost could be revived to life after dwelling in permafrost for many tens of millenia ${ }^{5}$.

Investigation of the molecular mechanisms that allow species to enter suspended animation was greatly facilitated by the finding that $C$. elegans, a well-established genetic model organism, can employ a metabolically quiescent stage known as a dauer larva to enter cryptobiosis $^{24}$. It has been shown that $C$. elegans possess a general program to survive different kinds of abiotic stress ${ }^{3,4}$. However, so far, it has only remained possible to maintain larvae in this state for relatively short periods in the laboratory without significant reductions in viability.

Here, we show that desiccation followed by freezing allows dauer larvae to retain full viability after prolonged periods in cryptobiosis. In parallel, we further investigated reanimated animals from permafrost. Precise radiocarbon dating indicates that they remained in cryptobiosis for about 46,000 years, since late Pleistocene. Genome assembly and morphological analysis define that these nematodes belong to new species (Panagrolaimus kolymaensis). We show that $P$. kolymaensis utilise similar mechanisms as $C$. elegans to survive desiccation and freezing under laboratory conditions. Our findings suggest nematodes possess adaptive mechanisms to survive in suspended animation for an indefinite time.

\section{C. elegans dauer larvae can remain in a cryptobiotic state for prolonged periods of time}

Our previous work on C. elegans dauer larvae led to the identification of several biochemical pathways that are required for entry into cryptobiosis ${ }^{3,4,10}$. This process is facilitated by exposure to mild desiccation ( $98 \% \mathrm{RH}$, relative humidity, 4 days) known as preconditioning ${ }^{10}$ 11 in which the transcriptome, proteome, and metabolic pathways undergo a specific

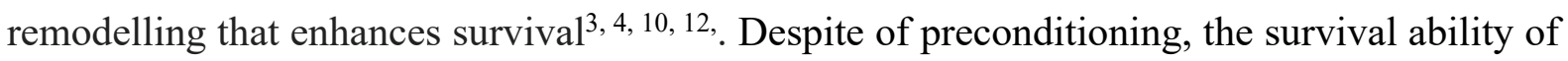
desiccated dauer larvae at room temperature declines very rapidly, with most larvae dead after around 10 days (Fig.1a). Direct freezing at $-80^{\circ} \mathrm{C}$, on the other hand, leads to almost instant death of the animals ${ }^{4}$. To test whether combining these conditions could extend the viability of 
dauer larvae, we transferred the desiccated larvae to $-80^{\circ} \mathrm{C}$. Remarkably, under these conditions, there was no significant decline in viability even after 480 days (Fig. 1a). Moreover, after thawing, the animals resumed reproductive growth and produced progeny in numbers similar to those of animals kept under control conditions (Fig. 1b). Since no reduction in survival has been observed at any point in extended experiments, these results suggest that the combination of anhydrobiosis and freezing can prolong the survival and reproductive ability of dauer larvae indefinitely.

\section{Discovery of reanimated nematodes from Permafrost: site and radiocarbon dating}

Our finding that $C$. elegans dauer larvae can remain viable for apparently indefinite periods following desiccation and freezing is surprising given that these nematodes are normally found in mild climatic zones. This ability, however, must be present in species that are exposed to harsher environment, for instance in polar regions or permafrost (perennially frozen sediments). Intensive research during the last decades has demonstrated that permafrosts are indeed unique ecosystems that preserve life forms at sub-zero temperatures over thousands of years ${ }^{13,14,15,1617,18}$. Interestingly, the possibility to exploit permafrost as a source for reanimating multicellular animals was already recognized in 1930s. P.N. Kapterev, as a GULAG prisoner, found a viable cladoceran crustacean Chydorus sphaericus preserved in the Transbaikalian permafrost for presumably several thousand years ${ }^{19,20}$. Unfortunately, this observation remained unnoticed for many decades.

It is well known that several species of Panagrolaimus can undergo several forms of cryptobiosis, namely anhydrobiosis (life without water) and cryobiosis (life at freezing) ${ }^{10,11 \text {, }}$ $21,22,23,24$. As mentioned above, in 2018 we showed that nematodes from the Siberian permafrost with morphologies consistent with the genera Panagrolaimus and Plectus could be reanimated thousands of years after they had been frozen in the permafrost ${ }^{5}$. Several viable nematode individuals were found in two out of more than 300 permafrost samples of different ages and genesis collected by researchers from the Soil Cryology Lab, Pushchino, Russia, in the course of perennial paleo-ecological expeditions carried out in the coastal sector of the northeastern Arctic $^{17}$. Detailed descriptions of the study site (outcrop Duvanny Yar, Kolyma River, Fig.2a), sampling and revitalizing procedures are provided in Supplementary Information. Like other late Pleistocene permafrost formations in the northeastern Arctic, Duvanny Yar comprises permanently frozen ice-rich silt deposits riddled with large polygonal ice wedges and divided by them into mineral blocks ${ }^{25,26}$ (Fig.2b). The sediments include sandy alluvial layers, peat lenses, buried paleosols and Pleistocene rodent burrows (Fig.2c). The burrow (P-1320), in which Panagrolaimus nematodes were found (Fig.2d), was taken from the frozen outcrop wall 
about $40 \mathrm{~m}$ below the surface and about $11 \mathrm{~m}$ above river water level in undisturbed and never thawed late Pleistocene permafrost deposits. The fossil burrow left by arctic gophers of the genus Citellus consisted of an entrance tunnel and a large nesting chamber up to $25 \mathrm{~cm}$ in diameter ${ }^{25}$.

The sterility of permafrost sampling and age of cultivated biota were discussed in detail in several reviews ${ }^{27,13,28}$. Based on previous reports, the age of the organisms found in a burrow is equal to the initial freezing time and corresponds to the age of organic matter conserved in the syncryogenic sediments. This makes it possible to use radiocarbon dating of organic matter to establish the age of organisms. We performed accelerator mass spectrometry (AMS) radiocarbon analysis of plant material obtained from studied borrow P-1320, and determined a direct ${ }^{14} \mathrm{C}$ age of $44,315 \pm 405 \mathrm{BP}$ (Institute of Geography, RAS; sample IGAN ${ }_{\text {AMS }}$ 9137). Calibrated age range is $45,839-47,769$ cal BP (95.4\% probability) (Fig.S1).

\section{Reanimated animals belong to a novel parthenogenetic species Panagrolaimus kolymaensis sp.nov.}

The revived animal was cultivated in the laboratory for over 100 generations and initially described as Panagrolaimus aff. detritophagus 5 based on morphology. We conducted a morphological analysis of the revived animal (Fig.3, S2, Table S1; See details of analysis in SI), which confirmed unambiguously that the animal belongs to the genus of Panagrolaimus, in agreement with a previous phylogenetic analysis of the $18 \mathrm{~S}$ ribosomal RNA sequence 5 . However, due to the morphological uniformity of Panagrolaimus, unusual even for nematodes, morphology and molecular analysis of a single ribosomal RNA sequence is insufficient to describe a species. We found the species to be parthenogenetic, which further complicates description under many species concepts.

To obtain comprehensive molecular data for determining the species using phylogenomics, we generated a high-quality genome assembly. Using PacBio HiFi sequencing, we generated $84 \mathrm{X}$ coverage in long reads (average length 14,425 bp). Our analysis of repeat and gene content is described in Supplementary Table 3. K-mer analysis of the reads clearly indicated that this animal has a triploid genome (Fig.4a), similar to other parthenogenetic Panagrolaimus species ${ }^{29}$. Despite the challenges that a triploid genome poses for assembly, we obtained a highly contiguous contig assembly of the three pseudohaplotypes that comprise a total of $\sim 266 \mathrm{Mb}$ and thus have a similar genome size as other parthenongenetic Panagrolaimus species $^{29}$. The contig N50 value of all three pseudohaplotypes is $3.8 \mathrm{Mb}$. Since the three pseudohaplotypes exhibited a noticeable degree of divergence, we further investigated their homology by using the apparent homeologs in our gene predictions to align long continuous 
contigs based on micro synteny (Fig. 4b). Links between the contigs clearly shows the triploid pattern of the genome.

To place the species in the genus Panagrolaimus, we generated an initial phylogeny using the $18 \mathrm{~S}$ and $28 \mathrm{~S}$ genes using maximum likelihood before conducting a broader multigene phylogenomic analysis using a concatenated, partitioned alignment of 60 genes and a coalescence-based approach using a broader set of 12,295 gene trees (see supplementary methods, Supplementary Table 4 and 5). These analyses retrieve the novel species as sister to all other sequenced Panagrolaimus species, but as an ingroup to Propanagrolaimus ${ }^{30}$ (Fig.4c; Fig.S3b-c). Thus, the phylogenetic placement provides strong evidence that this animal belongs to a novel species. Supporting this, there is substantial sequence divergence between this novel species, Panagrolaimus sp. PS1159 and Panagrolaimus sp. ES5, estimated in our concatenated alignment to be on average 2.06 and 2.11 amino acid substitutions per site, respectively. This divergence is consistent with previous data on ages of Panagrolaimus nematodes ${ }^{29}$, and more broadly with the divergence of nematodes in general, which can be hyper-diverse ${ }^{31,32}$. Our data also contradicts the assumption that parthenogenesis is monophyletic in the Panagrolaimus genus $^{29}$ (Fig.3c). Based on the Kolyma River location where the animal was unearthed, we propose the following taxonomic classification and species name:

Phylum Nematoda Potts, 1932

Class Chromadorea Inglis 1983

Suborder Tylenchina Thorne, 1949

Family Panagrolaimidae Thorne, 1937

Panagrolaimus kolymaensis sp. nov.

C. elegans dauer larvae and $P$. kolymaensis utilize similar mechanisms to enter and remain in cryptobiotic state for prolonged periods

The high-quality genome of $P$. kolymaensis allowed us to compare its molecular toolkit for cryptobiosis with that of C. elegans $3,4,10,12$. We used orthology clustering and phylogenetics to investigate whether the genome of $P$. kolymaensis contains genes previously implicated in cryptobiosis in C. elegans. Our analysis showed that the $P$. kolymaensis genome encodes orthologs to a $C$. elegans trehalose phosphate synthase gene (tps-2) and to a trehalose phosphatase gene (gob-1) (Fig.S4c, supplementary file Orthology analysis). Furthermore, we found orthologs to all C. elegans enzymes required for polyamine biosynthesis, the TCA cycle, glycolysis, gluconeogenesis, and glyoxylate shunt (Fig.S4c, supplementary file Orthology analysis) suggesting that $P$. kolymaensis possesses a similar but not identical molecular tool kit as $C$. elegans to survive cryptobiosis. 
To survive extreme desiccation, C. elegans dauer larvae need to be first preconditioned at high relative humidity $(98 \% \mathrm{RH})$ for 4 days ${ }^{10}$. During preconditioning, dauer larvae upregulate trehalose biosynthesis that ensures their survival to harsh desiccation ${ }^{10,12}$. We therefore tested whether preconditioning also facilitates survival of $P$. kolymaensis. Although a small proportion of $P$. kolymaensis individuals survive harsh desiccation and freezing without preconditioning (Fig.5a), similar to other panagrolaimid species ${ }^{11}$, mixed populations of $P$. kolymaensis survive harsh desiccation significantly longer $(\mathrm{p}<0.0001)$ following preconditioning (Fig.5a). Similarly, preconditioning and desiccation further enhanced survival of $P$. kolymaensis following freezing $\left(-80^{\circ} \mathrm{C}\right)$. Furthermore, like C. elegans, P. kolymaensis upregulate trehalose levels up to 20 -fold upon preconditioning (Fig.5b). We previously reported that, to upregulate trehalose levels upon preconditioning, C. elegans dauer larva dissipate their fat reserves (triacylglycerols) by activating the glyoxylate shunt and gluconeogenic pathway ${ }^{25}$. Upon preconditioning, we found that triacylglyceride (TAG) levels are significantly decreased in P. kolymaensis (Fig.S5a\&b). To further investigate whether the acetyl-CoA derived from degradation of TAGs culminate in trehalose, we applied the previously developed method of metabolic labelling with ${ }^{14} \mathrm{C}$-acetate in combination with $2 \mathrm{D}$ $\mathrm{TLC}^{4,25}$. As shown in Fig.5c, preconditioning led to a huge increase of radioactivity in trehalose and also to a lesser extent in some amino acids (glycine/serine, phenylalanine; panels c and d). Interestingly, $P$. kolymaensis displayed an additional spot (Fig.5d, enumerated as 7 ), that was not found in C. elegans ${ }^{25}$, which we identified as trehalose-6-phosphate (Fig.S5c-h), an immediate precursor of trehalose, using mass spectrometry. Thus, in order to resist harsh desiccation, similar to C. elegans, P. kolymaensis utilizes glyoxylate shunt and consequently acetate derived from TAGs to synthesize trehalose. Detection of the immediate precursor (trehalose-6-phosphate) suggests the flux of metabolites is intense in the latter.

Our findings indicate that by adapting to survival of the cryptobiotic state for short time frames in environments like permafrost, some nematode species gained the potential for individual worms to remain in the state for geological timeframes. This raises the question whether there is an upper limit to the length of time an individual can remain in the cryptobiotic state, potentially only limited by drastic changes to the environment such as strong fluctuations in ambient temperature, natural radioactivity or other abiotic factors. These findings have implications for our understanding of evolutionary processes, since generation times could be stretched from days to millennia, species ages might be much longer than anticipated, and longterm survival of individuals of species can lead to the refoundation of otherwise extinct lineages. Finally, understanding the precise mechanisms of long-term cryptobiosis and cues that lead to 
successful revivals can inform new methods for long term storage of cells and tissues and their transportation in space. 


\section{Methods}

\section{Materials and $C$. elegans strains}

[1-14C] -acetate (sodium salt) from Hartmann Analytic (Braunschweig, Germany). All other chemicals were purchased from Sigma-Aldrich (Taufkirchen, Germany). The Caenorhabditis Genetic Centre (CGC) provided daf-2(e1370) and E. coli NA22 strains.

\section{Genomic DNA isolation from Panagrolaimus kolymaensis nematodes}

P. kolymaensis nematodes were grown on several plates of NGM agar plated with E. Coli NA22 bacteria at $20^{\circ} \mathrm{C}$. Worms were collected from the plates, washed with water at least three to five times by centrifugation at $1000 \mathrm{~g}$ to remove any residual bacteria and any debris. The worm pellet was dissolved in 5 volumes of worm lysis buffer $(0.1 \mathrm{M}$ Tris- $\mathrm{HCl} \mathrm{pH}=8.5,0.1 \mathrm{M}$ $\mathrm{NaCl}, 50 \mathrm{mM}$ EDTA $\mathrm{pH}=8.0$ ) and distributed in $1.5 \mathrm{ml}$ of microcentrifuge tubes. These tubes are incubated at $-80 \mathrm{C}$ for 20 minutes. $100 \mathrm{ul}$ of Proteinase ' $\mathrm{K}$ ' $(20 \mathrm{mg} / \mathrm{ml})$ was added to each tube and they are incubated at $60 \mathrm{C}$ overnight. $625 \mathrm{ul}$ of cold GTC buffer (4M Guanidinium Thiocynate, $25 \mathrm{mM}$ Sodium citrate, $0.5 \%$ (v/v) N-lauroylsarcosine, 7\%(v/v) Beta Mercaptoethanol) was added to the tube, incubated on ice $30 \mathrm{~min}$ and mixed by inverting every 10 min. 1 volume of phenol-chloroform-isoamyl alcohol $(\mathrm{pH}=8)$ was added to the lysate and mixed by inverting the tube 10-15 times. Tubes were centrifuged for $5 \mathrm{~min}$ at $10,000 \mathrm{~g}$ at $4{ }^{\circ} \mathrm{C}$ to separate the phases. The upper aqueous phase was carefully collected into a fresh tube. One volume of fresh chloroform was added and mixed by inverting the tubes for 10-15 times and centrifuged for $5 \mathrm{~min}$ at $10,000 \mathrm{~g}$ at $4^{\circ} \mathrm{C}$ to separate the phases. One volume of cold $5 \mathrm{M} \mathrm{NaCl}$ was added, mixed by inverting the tubes and incubated on ice for $15 \mathrm{~min}$. After incubation these tubes were centrifuged for $15 \mathrm{~min}$ at $12,000-16,000 \mathrm{~g}$ at $4{ }^{\circ} \mathrm{C}$. The supernatant containing the nucleic acids were slowly transferred into a fresh tube. One volume of isopropanol was added to the tube, inverted few times and incubated on ice for 30 minutes. After incubation, the tubes were centrifuged at $3000 \mathrm{~g}$ for $30-45 \mathrm{~min}$ at $25^{\circ} \mathrm{C}$ and the supernatant was discarded without disturbing the pellet. The pellet was washed twice with $1 \mathrm{ml}$ of $70 \%$ ethanol, tubes were centrifuged at $3000 \mathrm{~g}$ for $5 \mathrm{~min}$ and supernatant was discarded and incubated at $37^{\circ} \mathrm{C}$ for 10-15 min to dry the pellet. The pellet was resuspended carefully in TE buffer. The quality and length of the high-molecular weight (HMW) genomic DNA (gDNA) was analyzed with pulse field gel electrophoresis. 


\section{Genome sequencing and assembly}

Long insert library has been prepared as recommended by Pacific Bioscienes according to the protocol 'Procedure \& Checklist-Preparing gDNA Libraries Using the SMRTbell® Express Template Preparation Kit 2.0'. In summary, RNAse treated HMW gDNA has been sheared to $20 \mathrm{~Kb}$ fragments on the MegaRuptor ${ }^{\mathrm{TM}}$ device (Diagenode) and $10 \mu \mathrm{g}$ sheared gDNA have been used for library preparation. The PacBio SMRTbell ${ }^{\mathrm{TM}}$ library was size selected in two fractions $(9-13 \mathrm{~kb},>13 \mathrm{~kb})$ using the BluePippin ${ }^{\mathrm{TM}}$ device with cassette definition of $0.75 \% \mathrm{DF}$ MarkerS1 3-10 kb improved Recovery. The second fraction of the size selected library has been loaded with $95 \mathrm{pM}$ on plate on one Sequel SMRT cell (8M). Sequel polymerase 2.0 has been used in combination with the v2 PacBio sequencing primer and the Sequel sequencing kit 2.0EA, runtime was 30 hours. We created PacBio CCS reads from the subreads.bam file using PacBio's ccs command linetool (version4.2.0). We obtained 8.5Gb hiqh quality CCS reads (HiFi reads) with a N50 of $14.4 \mathrm{~Kb}$. We ran HiCanu (version 2.2) ${ }^{33}$ to create the contig assembly. Blobtools (version 1.1.1,CITE) ${ }^{34}$ was used to identify and remove bacterial contigs. The final triploid contig assembly consists of 856 contigs has a N50 of $3.82 \mathrm{Mb}$ and a size of $266 \mathrm{Mb}$. The mitochondrial genome was created with the mitoHifi pipeline (version $2^{35}$ ) based on the assembled contigs and the closely related reference mitochondrial genome of Panagrellus redivivus (strain: PS2298/MT8872, ENAaccession: AP017464). The mitoHifi pipeline identified 49 mitochondrial contigs ranging from $13-32 \mathrm{~Kb}$. The final annotated circular mitochondrial genome has length of $17467 \mathrm{bp}$.

To identify pseudohaplotypes in the $P$. kolymanensis sp. nov genome assembly, we selected the longest isoform of each predicted protein-coding gene in our assembly and in the C. elegans genome (downloaded from WormBase Parasite, release WBPS15) using AGAT (version 0.4.0) and clustered them into orthologous groups (OGs) using OrthoFinder (version 2.5.2). We identified OGs that contained three Panagrolaimus sequences (i.e. groups that were present as single-copy in all three pseudohaplotypes) and used these to identify trios of multi-megabase size contigs derived from the three pseudohaplotypes. We visualized synteny between the three pseudohaplotypes using Circos to plot the positions of each homeolog (version 0.69-8).

\section{Genome annotation}

We used RepeatModeler 1.0.8 (http://www.repeatmasker.org/ ) with parameter '-engine ncbi' to create a library of repeat families. This library was used with RepeatMasker 4.0.9 to softmask the Panagrolaimus genome. To annotate genes, we cross mapped protein models from 
an existing Panagrolaimus as external evidence in the Augustus based pipeline ${ }^{36}$. We evaluated the completeness of our predictions using BUSCO on the gVolante web interface.

\section{Orthology analysis}

We conducted a gene orthology analysis using genomic data from Panagrolaimus kolymaensis, the plectid nematode species from the permafrost, as well as genomic data from WormBase Parasite (https://parasite.wormbase.org; accessed 17/12/2020): Caenorhabditis elegans, Diploscapter coronatus, Diploscapter pachys, Halicephalobus mephisto, Panagrellus redivivus, Panagrolaimus davidi, Panagrolaimus sp. ES5, Panagrolaimus sp. PS1159, Panagrolaimus superbus, Plectus sambesii, and Propanagrolaimus sp. JU765. For plectids, genomic resources are scarce. We therefore added transcriptome data of Plectus murrayi, Anaplectus granulosus, Neocamacolaimus parasiticus, and Stephanolaimus elegans. The latter three transcriptomes were kindly provided by Dr. Oleksandr Holovachov (Swedish museum of natural history). The Anaplectus granulosus, and Neocamacolaimus parasiticus transcriptomes have been published ${ }^{37,38}$. All three transcriptomes were assembled de novo with Trinity ${ }^{39}$. The exact procedures are described in the respective publications ${ }^{37,38}$. The Stephanolaimus elegans transcriptome was assembled in the same way as described for Neocamacolaimus parasiticus. The Plectus murrayi transcriptome was built from raw reads deposited at NCBI (https://sradownloadb.be-md.ncbi.nlm.nih.gov/sos2/sra-pub-run-13/SRR6827978/SRR6827978.1;

accessed 22.12.2020). The transcriptome was assembled using Galaxy Trinity version $2.9 .1^{40,39}$ using all default options and including in silico normalization of reads before assembly. Transdecoder (conda version 5.5.0) ${ }^{41}$ was used to translate to amino acid sequence. Identical reads were removed with cd-hit version 4.8.1 $1^{42,43}$, and the Trinity get_longest_isoform_seq_per_trinity_gene.pl command ${ }^{41}$ (Trinity conda version 2.8.5) (Anaconda Software Distribution, Conda, Version 4.9.2, Anaconda, Nov. 2020) was used, to remove shorter isoforms. Amino acid translated longest isoforms from genomic data were extracted with AGAT (Dainat, https://www.doi.org/10.5281/zenodo.3552717) from genome assembly FASTA files and genome annotation GFF3 files, using the agat_convert_sp_gxf2gxf.pl command to convert all GFF3 files to the required GFF3 format, and the agat_sp_keep_longest_isoform.pl as well as the agat_sp_extract_sequences.pl commands to extract amino acid translated FASTA files. The headers and names of all FASTA files were modified to allow for simple species assignment of each sequence in subsequent analysis. The orthology analysis was conducted with OrthoFinder v. 2.5. ${ }^{144,45}$ using default settings. For genes of interest, we constructed alignments with MAFFT v. $7.475^{46}$ using the 
localpair and maxiterate (1000) functions. We removed spurious sequences and areas that were not well aligned with Trimal v. 1.4.rev $22^{47}$ (procedure stated in supplementary file Orthology analysis below each phylogeny). We then ran phylogenetic analysis with Iqtree2 v. 2.0.64, with -bb 1000 option, testing the model for each analysis (models eventually used stated in supplementary file Orthology analysis). We also checked for PFAM domains using Interproscan v. 5.50-84.049. Part of the analysis was performed on the HPC RRZK CHEOPS of the Regional Computing Centre (RRZK) of the University of Cologne. The phylogenies were visualized with Dendroscope $3.7 .6^{50}$ and figures were created with Inkscape \{https://inkscape.org\}.

\section{Phylogenomics}

Sequences of $18 \mathrm{~S}$ and $28 \mathrm{~S}$ genes from 44 taxa across the Propanagrolaimus, Panagrolaimus, Panagrellus and Halicephalobus genera were aligned (MAFFT L-INS-I v7.475) ${ }^{46}$, concatenated $^{51}$ and used to infer a species tree using maximum likelihood via (IQTREE) ${ }^{52}$ and partitioned by best-fit models of sequence evolution for both ${ }^{53}$. Nodal support was determined using 1000 bootstrap pseudoreplicates. A further 60 genes from 101 taxa were used to confirm the taxonomic position using the supermatrix concatenation methods outlined above. Given the limitations of differential gene sampling, we expanded our phylogenomic analyses to include a coalescence approach using 12,295 ML gene trees inferred for orthogroups containing the target animal. Instances of multiple genes per species per group were treated as paralogs/orthologs and analysed using ASTRAL-Pro ${ }^{54}$.

\section{Desiccation survival assay}

C. elegans dauer larvae desiccation assays were performed as described in ${ }^{10}$ Panagrolaimus kolymaensis desiccation assays were performed with mixed populations of the worms.

\section{Exposure of nematodes to extreme environments}

Dauer larvae or Panagrolaimus kolymaensis nematodes were preconditioned and desiccated as described $^{24}$ and then transferred to elevated temperature of $34 \mathrm{C}$, freezing $\left(\begin{array}{lll}-80 & \mathrm{C}\end{array}\right)$ and anoxia. Anoxic environment was generated in a desiccation chamber at $60 \% \mathrm{RH}$ by flushing the Nitrogen gas into the chamber. The concentration of oxygen inside the chamber was monitored. After each timepoint they were rehydrated with $500 \mu \mathrm{l}$ of water for 2-3 hours. Rehydrated worms were transferred to NGM agar plates with E. Coli NA22 as food. Survivors 
were counted after overnight recovery. Each experiment was performed on two different days with at least two technical replicates.

\section{Trehalose quantification from nematode lysates}

Trehalose measurements were performed as described in ${ }^{12}$.

\section{Radiolabeling, metabolite extraction and 2D-TLC}

The above-mentioned procedures were performed according to previous reports ${ }^{4,12}$.

\section{Identification of trehalose-6-phosphate from TLC plates}

Normalised aqueous fractions from the non-preconditioned and preconditioned samples were separated by high performance thin layer chromatography (HPTLC), using 1-propanolmethanol-ammonia (32\%)-water $(28: 8: 7: 7 \mathrm{v} / \mathrm{v} / \mathrm{v} / \mathrm{v})$ as first, dried for $15 \mathrm{~min}$ and 1-butanolacetone-glacial acetic acid-water (35:35:7:23 v/v/v/v) second dimension respectively. Using the trehalose as a standard on both dimensions of the TLC, the regions of interest were scrapped out from the TLCs. The scraped-out silica was extracted with $10 \mathrm{ml}$ of $50 \%$ methanol twice. The fractions were combined, dried under vacuum and dissolved in $100 \mathrm{ul}$ of Ms mix solution containing 4:2:1 (Isopropanol:Methanol:Chloroform) with $7.5 \mathrm{mM}$ ammonium formate. Mass spectrometric analysis was performed on a Q Exactive instrument (Thermo Fischer Scientific, Bremen, DE) equipped with a robotic nanoflow ion source TriVersa NanoMate (Advion BioSciences, Ithaca, USA) using nanoelectrospray chips with a diameter of $4.1 \mu \mathrm{m}$. The ion source was controlled by the Chipsoft 8.3.1 software (Advion BioSciences). Ionization voltage was $+0.96 \mathrm{kV}$ in negative mode; backpressure was set at 1.25 psi. The temperature of the ion transfer capillary was $200^{\circ} \mathrm{C}$; S-lens RF level was set to $50 \%$. FT MS spectra were acquired within the range of $\mathrm{m} / \mathrm{z} 50-750$ at the mass resolution of $\mathrm{R} \mathrm{m} / \mathrm{z} 200=140000$; automated gain control (AGC) of $3 \times 10^{6}$ and with the maximal injection time of $3000 \mathrm{~ms}$. FT MS/MS spectra were acquired within the range of $\mathrm{m} / \mathrm{z} 50-750$ at the mass resolution of $\mathrm{R} \mathrm{m} / \mathrm{z} 200=140000$; automated gain control (AGC) of $3 \times 10^{4}$ and with the maximal injection time of $30 \mathrm{~s}$.

\section{Triacylglycerols measurement from Panagrolaimus kolymaensis lysates}

Non-preconditioned and preconditioned pellets were lysed in $200 \mathrm{ul}$ of isopropanol with 0.5 $\mathrm{mm}$ Zircornium beads twice for $15 \mathrm{~min}$. The lysates were centrifuged at $1300 \mathrm{~g}$ for $5 \mathrm{~min}$ and $20 \mathrm{ul}$ of the lysate was used for protein estimation. The supernatant was carefully collected 
without any debris and was dried in the desiccator. To dried samples 700 ul of 10:3 (v/v) MTBE/MeOH solution with internal standard (TG-d5 50:0) were added and left on a shaker for 1 hour at $1400 \mathrm{rpm}$ and $4^{\circ} \mathrm{C} .140 \mathrm{ul}$ of water was added and left on shaker for $15 \mathrm{~min}$ at $4^{\circ} \mathrm{C}$. These samples were centrifuged at $13400 \mathrm{rpm}$ for $15 \mathrm{~min}$ at $4^{\circ} \mathrm{C}$. The upper organic fraction was collected and transferred to $1.5 \mathrm{ml}$ glass vial and left for drying in the desiccator. The dried samples were reconstituted in appropriate volume of $300 \mathrm{ul}$ of 4:2:1 (Isopropanol: Methanol: Chloroform) and volume corresponding to $1 \mu \mathrm{g}$ protein was used for injection.

LC-MS/MS analysis was performed on a high-performance liquid chromatography system (Agilent 1200 HPLC) coupled to a Xevo G2-S QTof (Waters). The samples were resolved on a reverse phase C18 column (Cortecs C18 2.7um from Waters) with 50:50:0.1:1\% (Water:methanol:formic acid: $1 \mathrm{M}$ Ammonium formate) and 25:85:0.1:1\% (Acetonitrile:Isopropanol:Formic acid:1M Ammonium formate) as mobile phase. The following gradient program was used: Eluent B from $0 \%$ to $100 \%$ within $12 \mathrm{~min} ; 100 \%$ from $12 \mathrm{~min}$ to $17 \mathrm{~min} ; 0 \%$ from $17 \mathrm{~min}$ to $25 \mathrm{~min}$. The flow rate was set at $0.3 \mathrm{ml} / \mathrm{min}$. The samples were normalised according to the total protein concentration and the worm numbers. The TG species were extracted from Lipidmaps database and Skyline software was used to analyse the TG profile. 


\section{Author contributions}

AS, VG, PS, TK conceived and designed the study. AS, VG, TH, MP, AT, GH, MH, ER, PS and TK contributed to the original draft. AS performed isolation and cultivation of nematodes. VG performed desiccation survival assays, trehalose measurement, 2D-TLC of metabolites, trehalose-6-phosphate detection, combination of cryptobiosis experiments, genomic DNA isolation from Panagrolaimus kolymaensis and prepared the figures. MP performed genome assembly. TH conducted orthology analyses and single gene phylogenies, and worked on figures. AT provided morphological description, light and scanning electron microscopy. LS analyzed the genome assembly and created figures. GMH performed the phylogenetic analyses. ST performed triacylglycerols measurements, trehalose-6-phosphate detection with suggestions from Andrej Shevchenko. MH annotated supplementary table 3. PS performed genome annotation and supervised TH. EWM supervised MP and had overall responsibility for sequencing and assembly including funding it. 


\section{Acknowledgements}

VG is thankful to Andrej Shevchenko, members of Kurzchalia lab for helpful discussions and core facilities of MPI-CBG for assistance. We are grateful to Dr. S. Gubin for field study and sampling, our colleagues in Soil Cryology Lab, Pushchino and North-East Scientific Station in Chersky, Republic of Sakha (Yakutia) for their help and cooperation. The authors thank Long Read Team of the DRESDEN-concept Genome Center, DFG NGS Competence Center, part of the Center for Molecular and Cellular Bioengineering (CMCB), Technische Universität Dresden and MPI-CBG. GMH is funded by a UCD Ad Astra Fellowship. This work was supported by the Russian Foundation for Basic Research (19-29-05003-mk) to AS and ER. VG and TK financial support from the Volkswagen Foundation (Life? research grant 92847). PS and TH are supported by a DFG ENP grant to PS (DFG project 434028868). The authors are thankful to Richard Roy and Jens Bast for critical reading the manuscript. We thank Iain Pattern for suggestions in writing the manuscript. 


\section{References}

1. Keilin, D. The Leeuwenhoek Lecture - The problem of anabiosis or latent life: history and current concept. Proc. R. Soc. Lond. Ser. B - Biol. Sci. 150, 149-191 (1959).

2. Hibshman, J. D., Clegg, J. S. \& Goldstein, B. Mechanisms of Desiccation Tolerance: Themes and Variations in Brine Shrimp, Roundworms, and Tardigrades. Front. Physiol. 11, $592016(2020)$.

3. Erkut, C. et al. Molecular Strategies of the Caenorhabditis elegans Dauer Larva to Survive Extreme Desiccation. PLoS ONE 8, e82473 (2013).

4. Gade, V. R., Traikov, S., Oertel, J., Fahmy, K. \& Kurzchalia, T. V. C. elegans possess a general program to enter cryptobiosis that allows dauer larvae to survive different kinds of abiotic stress. Sci. Rep. 10, 13466 (2020).

5. Shatilovich, A. V. et al. Viable Nematodes from Late Pleistocene Permafrost of the Kolyma River Lowland. Dokl. Biol. Sci. 480, 100-102 (2018).

6. Cano, R. \& Borucki, M. Revival and identification of bacterial spores in 25- to 40-millionyear-old Dominican amber. Science 268, 1060-1064 (1995).

7. Shen-Miller, J. Sacred lotus, the long-living fruits of China Antique. Seed Sci. Res. 12, 131143 (2002).

8. Shmakova, L. et al. A living bdelloid rotifer from 24,000-year-old Arctic permafrost. Curr. Biol. 31, R712-R713 (2021).

9. Guidetti, R. \& Jönsson, K. I. Long-term anhydrobiotic survival in semi-terrestrial micrometazoans. J. Zool. 257, 181-187 (2002).

10.Erkut, C. et al. Trehalose Renders the Dauer Larva of Caenorhabditis elegans Resistant to Extreme Desiccation. Curr. Biol. 21, 1331-1336 (2011).

11.McGill, L. M. et al. Anhydrobiosis and Freezing-Tolerance: Adaptations That Facilitate the Establishment of Panagrolaimus Nematodes in Polar Habitats. PLOS ONE 10, e0116084 (2015).

12.Erkut, C., Gade, V. R., Laxman, S. \& Kurzchalia, T. V. The glyoxylate shunt is essential for desiccation tolerance in C. elegans and budding yeast. elife 5, (2016).

13.Gilichinsky, D. A. et al. Microbial Populations in Antarctic Permafrost: Biodiversity, State, Age, and Implication for Astrobiology. Astrobiology 7, 275-311 (2007).

14.Rivkina, E. et al. Earth's perennially frozen environments as a model of cryogenic planet ecosystems. Permafr. Periglac. Process. 29, 246-256 (2018). 
15.Sipes, K. et al. Eight Metagenome-Assembled Genomes Provide Evidence for Microbial Adaptation in 20,000- to 1,000,000-Year-Old Siberian Permafrost. Appl. Environ. Microbiol. 87, (2021).

16.Liang, R. et al. Genomic reconstruction of fossil and living microorganisms in ancient Siberian permafrost. Microbiome 9, 110 (2021).

17.Gilichinsky, D. A. \& Rivkina, E. M. PERMAFROST MICROBIOLOGY. 7 (2011).

18.Jansson, J. K. \& Taş, N. The microbial ecology of permafrost. Nat. Rev. Microbiol. 12, 414425 (2014).

19.Kapterev, P. N. Anabiosis in the conditions of permanent congelation. Bulletin de L'Academie des Sciences de L'URSS 6, 1073-1088 (1936).

20.Kapterev, P. New data on revitalization of organisms from perpetually frozen grounds. $C R$ Acad Sci URSS 20, 315-317 (1938).

21.Adhikari, B. N., Wall, D. H. \& Adams, B. J. Desiccation survival in an Antarctic nematode: molecular analysis using expressed sequenced tags. BMC Genomics 10, 69 (2009).

22.Tyson, T. et al. A molecular analysis of desiccation tolerance mechanisms in the anhydrobiotic nematode Panagrolaimus superbus using expressed sequenced tags. BMC Res. Notes 5, 68 (2012).

23.Thorne, M. A. S., Kagoshima, H., Clark, M. S., Marshall, C. J. \& Wharton, D. A. Molecular Analysis of the Cold Tolerant Antarctic Nematode, Panagrolaimus davidi. PLoS ONE 9, e104526 (2014).

24.Sandhove, J., Spann, N. \& Ristau, K. The Anhydrobiotic Potential of the Terrestrial Nematodes Plectus parietinus and Plectus velox: ANHYDROBIOSIS OF PLECTUS PARIETINUS AND P. VELOX. J. Exp. Zool. Part Ecol. Genet. Physiol. 325, 434-440 (2016).

25.Zanina, O. G., Gubin, S. V., Kuzmina, S. A., Maximovich, S. V. \& Lopatina, D. A. LatePleistocene (MIS 3-2) palaeoenvironments as recorded by sediments, palaeosols, and ground-squirrel nests at Duvanny Yar, Kolyma lowland, northeast Siberia. Quat. Sci. Rev. 30, 2107-2123 (2011).

26.Murton, J. B. et al. Palaeoenvironmental Interpretation of Yedoma Silt (Ice Complex) Deposition as Cold-Climate Loess, Duvanny Yar, Northeast Siberia: Palaeoenvironmental Interpretation of Yedoma Silt, Duvanny Yar. Permafr. Periglac. Process. 26, 208-288 (2015).

27.Abramov, A., Vishnivetskaya, T. \& Rivkina, E. Are permafrost microorganisms as old as permafrost? FEMS Microbiol. Ecol. 97, fiaa260 (2021). 
28.Gilichinsky, D. A., Wagener, S. \& Vishnevetskaya, T. A. Permafrost microbiology. Permafr. Periglac. Process. 6, 281-291 (1995).

29.Schiffer, P. H. et al. Developmental variations among Panagrolaimid nematodes indicate developmental system drift within a small taxonomic unit. Dev. Genes Evol. 224, 183-188 (2014).

30.Schiffer, P. H. et al. Signatures of the Evolution of Parthenogenesis and Cryptobiosis in the Genomes of Panagrolaimid Nematodes. iScience 21, 587-602 (2019).

31.Dey, A., Chan, C. K. W., Thomas, C. G. \& Cutter, A. D. Molecular hyperdiversity defines populations of the nematode Caenorhabditis brenneri. Proc. Natl. Acad. Sci. 110, 1105611060 (2013).

32.Cutter, A. D. Divergence Times in Caenorhabditis and Drosophila Inferred from Direct Estimates of the Neutral Mutation Rate. Mol. Biol. Evol. 25, 778-786 (2008).

33.Nurk, S. et al. HiCanu: accurate assembly of segmental duplications, satellites, and allelic variants from high-fidelity long reads. Genome Res. 30, 1291-1305 (2020).

34.Laetsch, D. R. \& Blaxter, M. L. BlobTools: Interrogation of genome assemblies. F1000Research 6, 1287 (2017).

35.Allio, R. et al. MitoFinder: Efficient automated large-scale extraction of mitogenomic data in target enrichment phylogenomics. Mol. Ecol. Resour. 20, 892-905 (2020).

36.Brůna, T., Hoff, K. J., Lomsadze, A., Stanke, M. \& Borodovsky, M. BRAKER2: automatic eukaryotic genome annotation with GeneMark-EP+ and AUGUSTUS supported by a protein database. NAR Genomics Bioinforma. 3, lqaa108 (2021).

37. Smythe, A. B., Holovachov, O. \& Kocot, K. M. Improved phylogenomic sampling of freeliving nematodes enhances resolution of higher-level nematode phylogeny. BMC Evol. Biol. 19, 121 (2019).

38.Ahmed, M., Adedidran, F. \& Holovachov, O. A draft transcriptome of a parasite Neocamacolaimus parasiticus (Camacolaimidae, Plectida). J. Nematol. 53, 1-4 (2021).

39.Grabherr, M. G. et al. Full-length transcriptome assembly from RNA-Seq data without a reference genome. Nat. Biotechnol. 29, 644-652 (2011).

40.Goecks, J., Nekrutenko, A., Taylor, J. \& Galaxy Team, T. Galaxy: a comprehensive approach for supporting accessible, reproducible, and transparent computational research in the life sciences. Genome Biol. 11, R86 (2010).

41.Haas, B. J. et al. De novo transcript sequence reconstruction from RNA-seq using the Trinity platform for reference generation and analysis. Nat. Protoc. 8, 1494-1512 (2013). 
42.Li, W. \& Godzik, A. Cd-hit: a fast program for clustering and comparing large sets of protein or nucleotide sequences. Bioinformatics 22, 1658-1659 (2006).

43.Fu, L., Niu, B., Zhu, Z., Wu, S. \& Li, W. CD-HIT: accelerated for clustering the nextgeneration sequencing data. Bioinformatics 28, 3150-3152 (2012).

44.Emms, D. M. \& Kelly, S. OrthoFinder: solving fundamental biases in whole genome comparisons dramatically improves orthogroup inference accuracy. Genome Biol. 16, 157 (2015).

45.Emms, D. M. \& Kelly, S. OrthoFinder: phylogenetic orthology inference for comparative genomics. Genome Biol. 20, 238 (2019).

46.Katoh, K. \& Standley, D. M. MAFFT Multiple Sequence Alignment Software Version 7: Improvements in Performance and Usability. Mol. Biol. Evol. 30, 772-780 (2013).

47.Capella-Gutierrez, S., Silla-Martinez, J. M. \& Gabaldon, T. trimAl: a tool for automated alignment trimming in large-scale phylogenetic analyses. Bioinformatics 25, 1972-1973 (2009).

48.Minh, B. Q. et al. IQ-TREE 2: New Models and Efficient Methods for Phylogenetic Inference in the Genomic Era. Mol. Biol. Evol. 37, 1530-1534 (2020).

49.Jones, P. et al. InterProScan 5: genome-scale protein function classification. Bioinformatics 30, 1236-1240 (2014).

50.Huson, D. H. \& Scornavacca, C. Dendroscope 3: An Interactive Tool for Rooted Phylogenetic Trees and Networks. Syst. Biol. 61, 1061-1067 (2012).

51.Kück, P. \& Meusemann, K. FASconCAT: Convenient handling of data matrices. Mol. Phylogenet. Evol. 56, 1115-1118 (2010).

52.Nguyen, L.-T., Schmidt, H. A., von Haeseler, A. \& Minh, B. Q. IQ-TREE: A Fast and Effective Stochastic Algorithm for Estimating Maximum-Likelihood Phylogenies. Mol. Biol. Evol. 32, 268-274 (2015).

53.Kalyaanamoorthy, S., Minh, B. Q., Wong, T. K. F., von Haeseler, A. \& Jermiin, L. S. ModelFinder: fast model selection for accurate phylogenetic estimates. Nat. Methods 14, 587-589 (2017).

54.Zhang, C., Scornavacca, C., Molloy, E. K. \& Mirarab, S. ASTRAL-Pro: Quartet-Based Species-Tree Inference despite Paralogy. Mol. Biol. Evol. 37, 3292-3307 (2020).

55.Reimer, P. J. et al. The IntCal20 Northern Hemisphere Radiocarbon Age Calibration Curve (0-55 cal kBP). Radiocarbon 62, 725-757 (2020).

56.Ranallo-Benavidez, T. R., Jaron, K. S. \& Schatz, M. C. GenomeScope 2.0 and Smudgeplot for reference-free profiling of polyploid genomes. Nat. Commun. 11, 1432 (2020). 
bioRxiv preprint doi: https://doi.org/10.1101/2022.01.28.478251; this version posted January 28, 2022. The copyright holder for this preprint (which was not certified by peer review) is the author/funder. All rights reserved. No reuse allowed without permission.

57.Kokot, M., Długosz, M. \& Deorowicz, S. KMC 3: counting and manipulating k-mer statistics. Bioinformatics 33, 2759-2761 (2017). 
Figure 1. C. elegans dauer larvae can remain in a cryptobiotic state for prolonged periods of time. a) Desiccated $d a f-2(e 1370)$ dauer larvae survive to freezing $\left(-80^{\circ} \mathrm{C}\right)$ for an extremely long period. Error bars indicate standard error of mean of two independent experiments with two technical replicates performed on two different days. b) Brood size of desiccated dauer larvae exposed to freezing remain similar to that non- desiccated dauer larvae. Average brood size is the mean of seven dauer larvae per each condition. Statistical comparison was performed by using non-parametric Kolmogorov-smirnov test n.s p $>0.05$. 


\section{Figure 2. Study site.}

a) Location of the Duvanny Yar outcrope on the Kolyma River, northeastern Siberia, Russia.

b) view of the upper part of outcrop composed of ice wedges and permafrost silty deposits. c) lithostratigraphic scheme of deposits, showing location of studied rodent borrow (red circle).

d) Fossil rodent burrow with herbaceous litter and seeds buried in permafrost deposits. $m$ a.r.l. $=$ meters above river level. 


\section{Figure 3. General morphology of Panagrolaimus kolymaensis sp. n., female.}

Scanning electron pictures (a, c), light microscopy photographs (e, f) and graphic presentations (b, d, g) of holotype: a, b) entire body, c, d) anterior ends, e) anterior body, f) perivulvar body region, g) tail. Abbreviations: 1.f. - lateral field, ov - ovary, pro - procorpus of the pharynx, t.b. - terminal bulb of the pharynx, u - uterus with eggs, v - vulva, v.p. - ventral pore. Scale bars: a, d, e, f, g $-20 \mu \mathrm{m}, \mathrm{b}-100 \mu \mathrm{m}, \mathrm{c}-2 \mu \mathrm{m}$. 
Figure 4. Genome assembly and phylogenomics reveals that the newly discovered Panagrolaimus kolymaensis species is triploid.

a) Kmer spectra of the Panagrolaimus kolymaensis PacBio HiFi data. Kmers of length 19 were counted using Jellyfish. b) Circos plot showing the triploid structure of the Panagrolaimus kolymensis genome. The relationship between eight contigs, representing $39.9 \mathrm{Mb}(15 \%)$ of the genome, is shown. Contig IDs are shown. Scale is in megabases (Mb). c) Inferred species tree for all taxa. The maximum likelihood tree inferred using a concatenated supermatrix (18S and $28 \mathrm{~S}$ genes) with bootstrap support values is displayed. All genera are represented as monophyletic clades. Panagrolaimus kolymaensis is highlighted in red and basal to all other Panagrolaimus taxa. Internal nodes, where all subsequent branches represent identical sequences, are displayed with a black star. 
Figure 5. C. elegans dauer larvae and P. kolymaensis utilize similar mechanisms to survive cryptobiosis.

a) Survival rate of $P$. kolymaensis nematodes to desiccation and freezing $\left(-80^{\circ} \mathrm{C}\right)$. Error bars indicate standard error of mean of two independent experiments with two technical replicates performed on two different days. Statistical comparison was performed using unpaired t test with Welch correction. n.s. $\mathrm{p}>0.05, * * * * \mathrm{p}<0.0001$. For desiccation (non-preconditioned) $\mathrm{n}$ $=289$, freezing (non-preconditioned) $\mathrm{n}=675$, desiccation (preconditioned at $98 \% \mathrm{RH}$ ) $\mathrm{n}=953$ and freezing (preconditioned at $98 \% \mathrm{RH}) \mathrm{n}=1295$. b) P. kolymaensis nematodes and daf2(e1370) dauer larvae upregulate trehalose levels upon preconditioning at 98\%RH. Error bars indicate standard error of mean of two independent experiments with three replicates performed on two different days. Statistical comparison was performed using two-way ANOVA with Holm-Sidak's multiple comparison test, $* * * * p<0.0001$. c-d) 2D-thin layer chromatography of ${ }^{14} \mathrm{C}$-acetate labelled metabolites from $P$. kolymaensis that were nonpreconditioned and preconditioned at $98 \% \mathrm{RH}$. Enumerated spots indicate trehalose (1), glucose (2), glutamate (3), glutamine (4), serine/glycine (5) and phenylalanine (6). Representative images from at least two independent experiments performed on two different days. 


\section{Figure S1. Calibration of a radiocarbon $\left({ }^{14} \mathrm{C}\right)$ date}

Radiocarbon date $(44,315 \pm 405$ BP) and calibrated age $(45,839-47,769$ cal BP) of plant material collected from buried borrow P-1320. Radiocarbon ages were converted to calendar age equivalents with the OxCal V.4.4 program using the IntCal20 calibration curve ${ }^{55}$. Pinkshaded area — radiocarbon date with standard deviation; grey-shaded area — radiocarbon date projection on the calibration curve with $95.4 \%$ probability. 


\section{Figure S2. Morphology of Panagrolaimus kolymaensis sp. n., female.}

Graphic presentations of holotype (a,b) and SEM pictures (c-i): a) anterior body, b) female reproductive branch, c-e) anterior end of three different female specimens, f) anterior part of the lateral ridge, g) vulva, h) ventral excretory/secretory pore, i) posterior body with anus and lateral ridge. Scale bars: a,b - $50 \mu \mathrm{m}$, c-e - $3 \mu \mathrm{m}, \mathrm{f}, \mathrm{g}, \mathrm{i}-10 \mu \mathrm{m}, \mathrm{h}-5 \mu \mathrm{m}$. 


\section{Figure S3. Phylogenies inferred for genes sets using both concatenation and coalescence} approaches.

a) The ploidy level was analysed with Smudgeplot v0.2.1 ${ }^{56}$. KMC version 3.1.0 ${ }^{57}$ was used to count the 21-mers in the PacBio CCS reads. Then we ran smudgeplot.py to determine the lower and upper coverage cut-offs. These were determined to be 14 and 380.21-mers with a coverage between 14 and 380 were filtered with kmc_tools. Then, we computed the k-mer pairs from the filtered 21-mers by running smudgeplot.py hetkmers. Finally, the produced a smudgeplot shows an estimated ploidy of 3. b) Species tree inferred for 12,295 gene trees using a coalescence approach. The species tree implemented using the coalescence approach with orthogroup gene trees is displayed. Novel species in this study are displayed in red. All nodes have a posterior probability of 1. c) Species tree inferred for 102 taxa. The maximum likelihood tree inferred using a concatenated supermatrix of 60 genes is displayed. Bootstrap values are only displayed for nodes with less than 100\% support. The platyhelminth Macrostum lignano serves as an outgroup for rooting. The Ancestral panagrolaimus, basal to all others within the Panagrolaimus genera, is highlighted in red. 
Figure S4. Combination of cryptobiotic states enhances survival of C. elegans dauer larvae.

a) Desiccated dauer larvae manifest enhanced survival rate to heat stress $\left(34^{\circ} \mathrm{C}\right)$. Error bars indicate standard deviation of two independent experiments with two technical replicates. b) Desiccated dauer larvae display enhances survival rate to anoxia. Error bars indicate standard deviation of two independent experiments with two technical replicates. Statistical comparison was performed by paired two tailed t-test. ${ }^{*} \mathrm{p}<0.05$. c) $P$. kolymaensis possesses $C$. elegans gene orthologs to enzymes required for TCA cycle, glyoxylate shunt, glycolysis, gluconeogenesis, trehalose synthesis, and polyamine synthesis. Black filled circles: Ortholog presence suggested by orthogroup clustering, phylogenetic analysis, and domain architecture. White filled circles: No ortholog found via current analysis. Coloured filled circles: Presence of $P$. kolymaensis gene(s), related to a number of C. elegans genes (all genes of same colour) that are all co-orthologous to that gene (those genes). Label: C. elegans enzyme names and orthogroup that contains that gene according to our orthogroup clustering 
Figure S5. $P$. kolymaensis reduces triacylglycerols (TAGs) levels and accumulates trehalose-6-phosphate upon preconditioning at $98 \% \mathrm{RH}$.

a) 1D-Thin layer chromatography of acetate labelled organic fractions of non-preconditioned (1) and preconditioned (2) P. kolymaensis. b) Mass spectrometric quantification of TAG levels of non-preconditioned (1) and preconditioned (2) P. kolymaensis. Error bars indicate standard deviation of two independent experiments with two technical replicates. Statistical analysis was performed using unpaired t-test with Welch correction $* * p<0.001$. c-d) nonpreconditioned and preconditioned mass spectrum of an empty region, e-f) spot 1 (trehalose), g-h) spot 7 (trehalose-6-phosphate) scraped out and extracted from the 2D-TLC. 


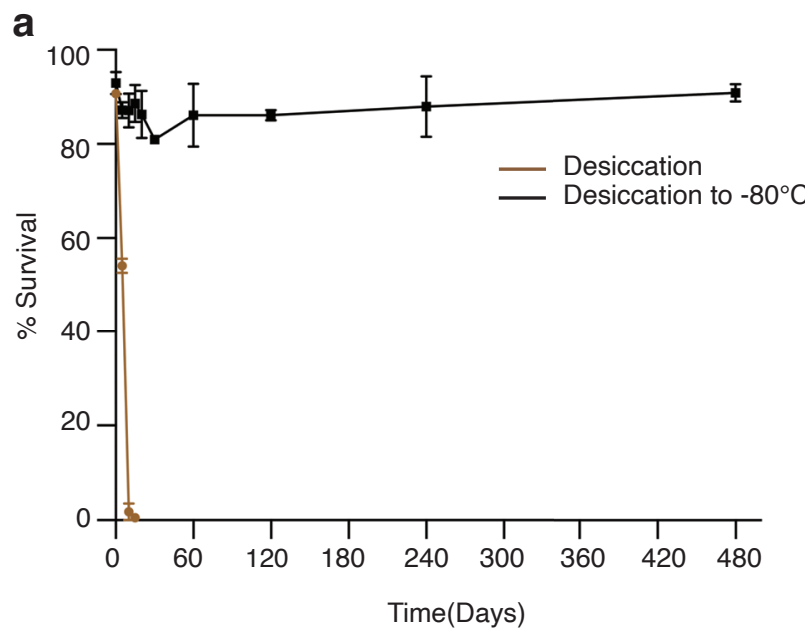

b

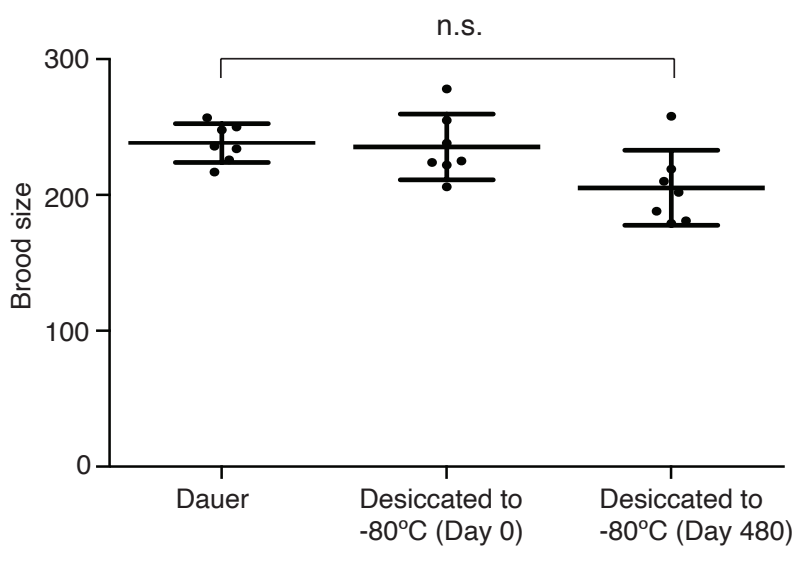

Figure 1

Shatilovich et al 

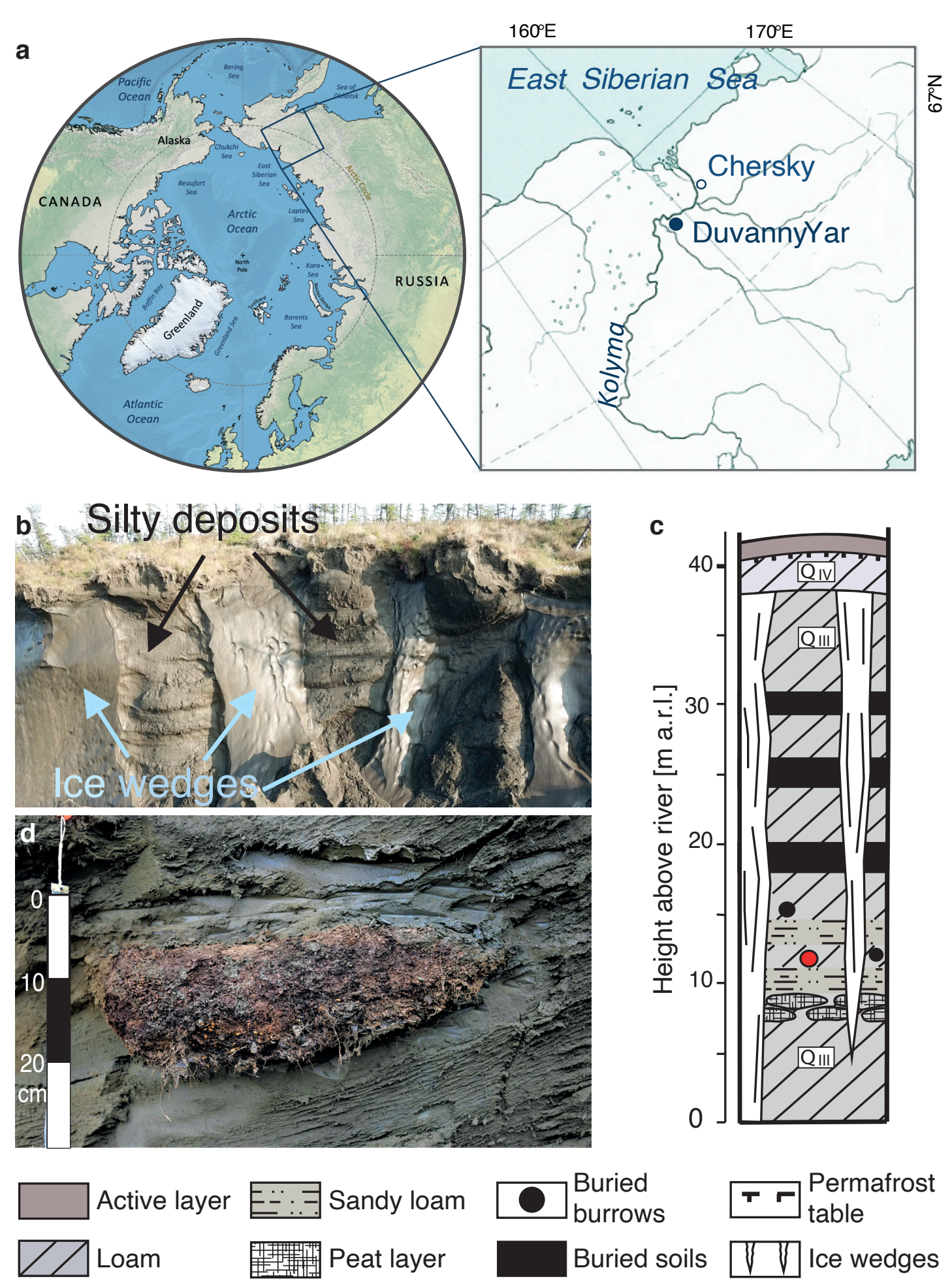

Figure 2

Shatilovich et al 

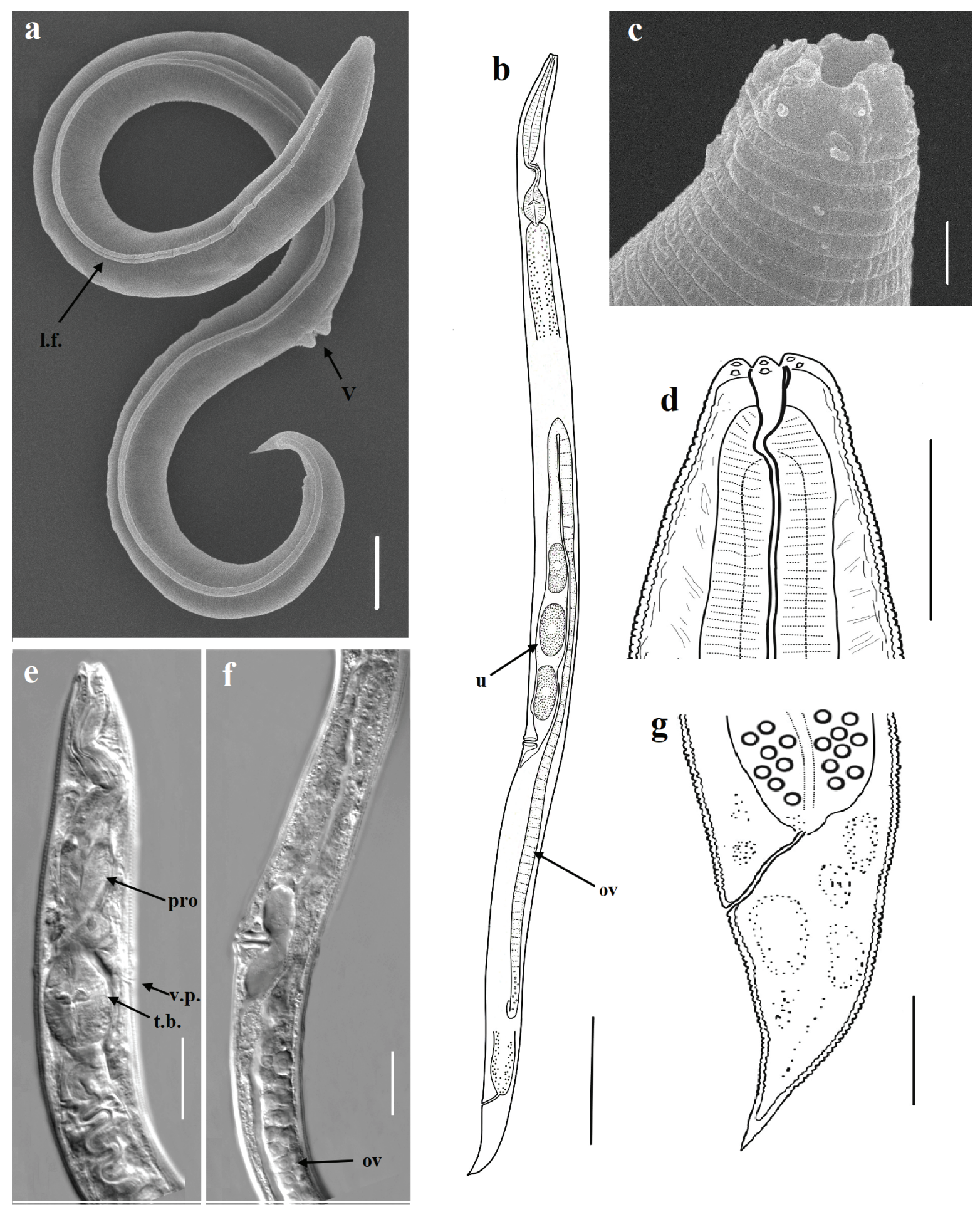

Figure 3

Shatilovich et al 
bioRxiv preprint doi: https://doi.org/10.1101/2022.01.28.478251; this version posted January 28, 2022. The copyright holder for this preprint (which was not certified by peer review) is the author/funder. All rights reserved. No reuse allowed without permission.

a

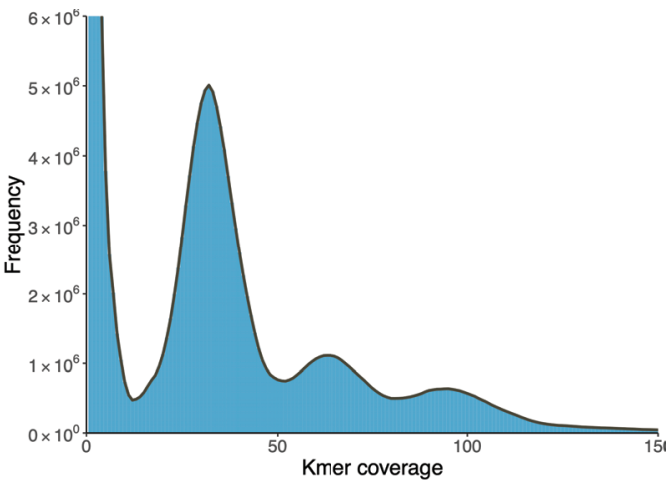

b

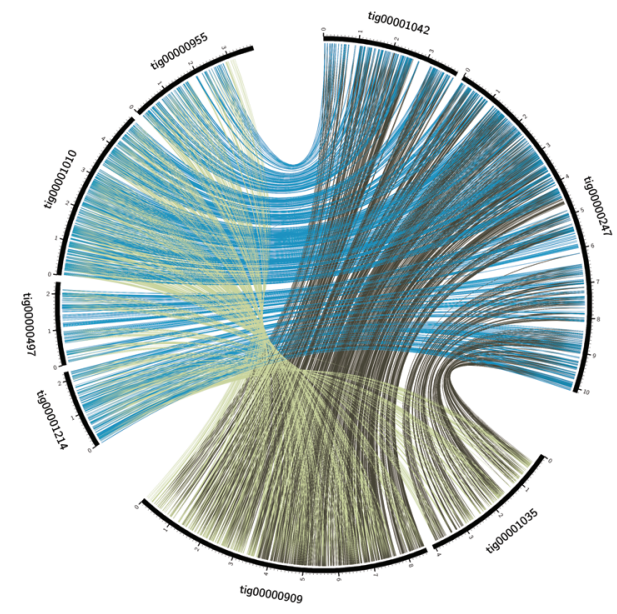

C

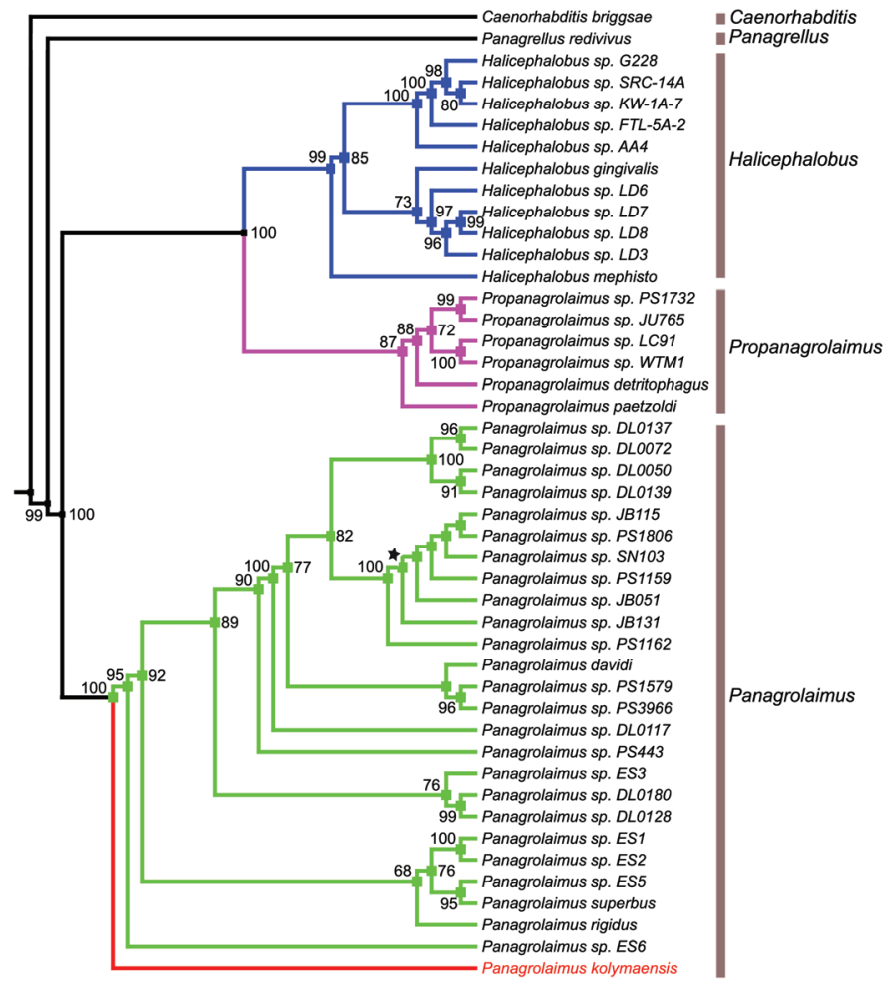

Figure 4

Shatilovich et al 

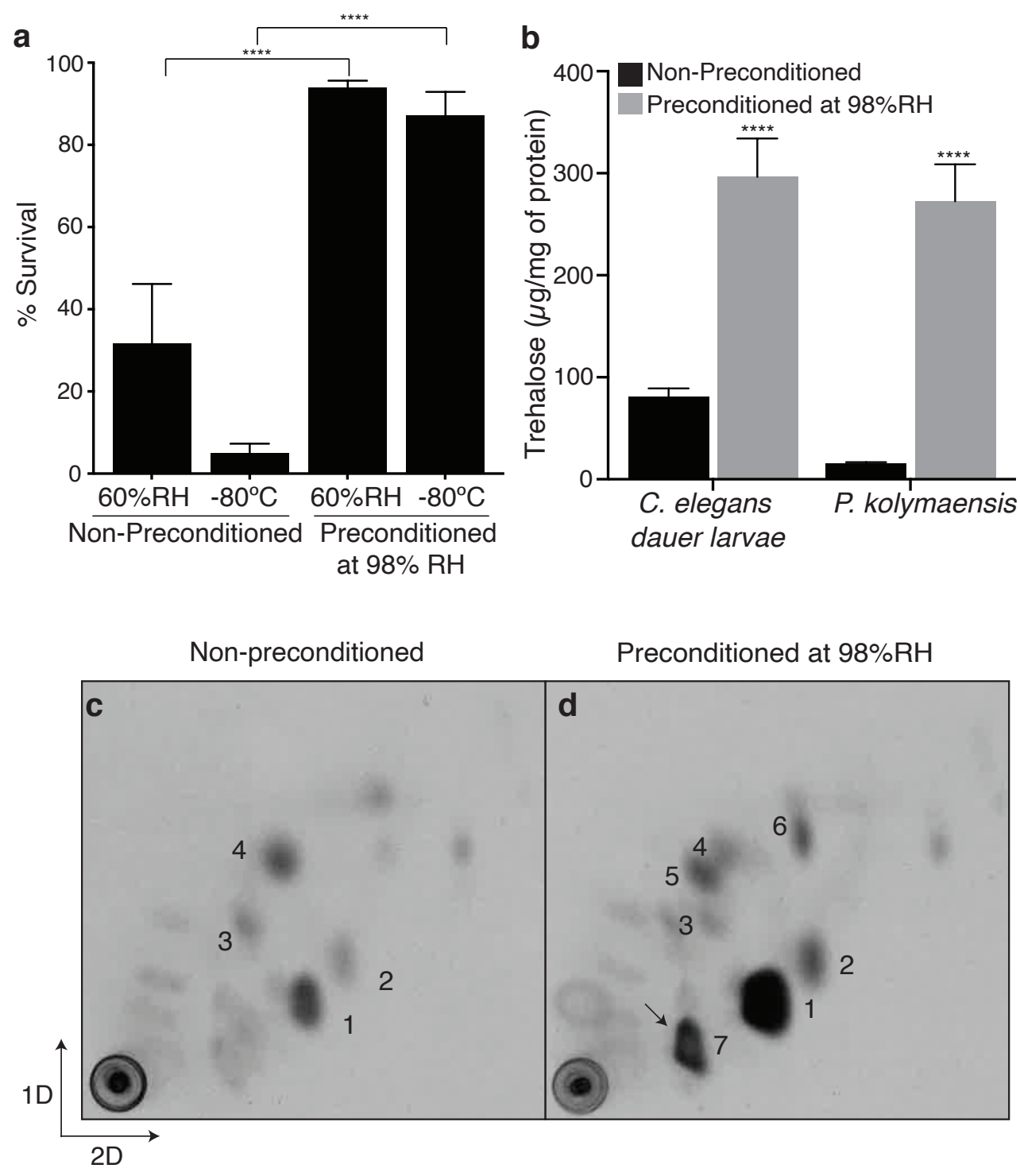

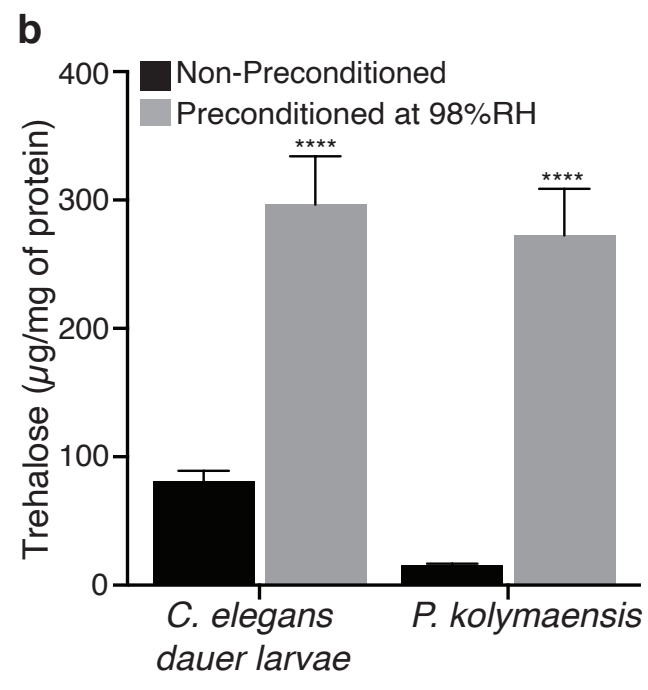

Figure 5

Shatilovich et al 


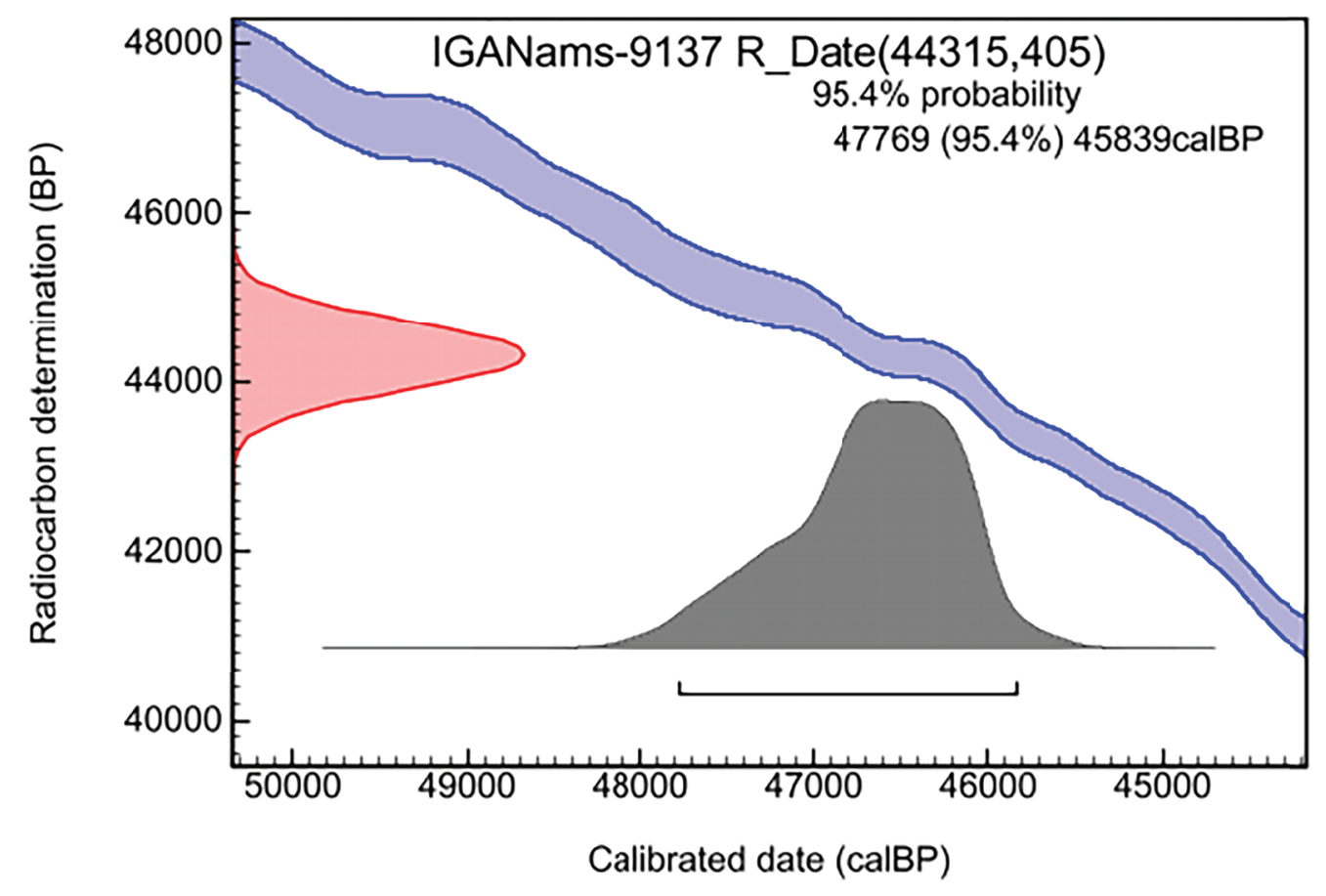

Figure S1

Shatilovich et al 
bioRxiv preprint doi: https://doi.org/10.1101/2022.01.28.478251; this version posted January 28, 2022. The copyright holder for this preprint (which was not certified by peer review) is the author/funder. All rights reserved. No reuse allowed without permission.
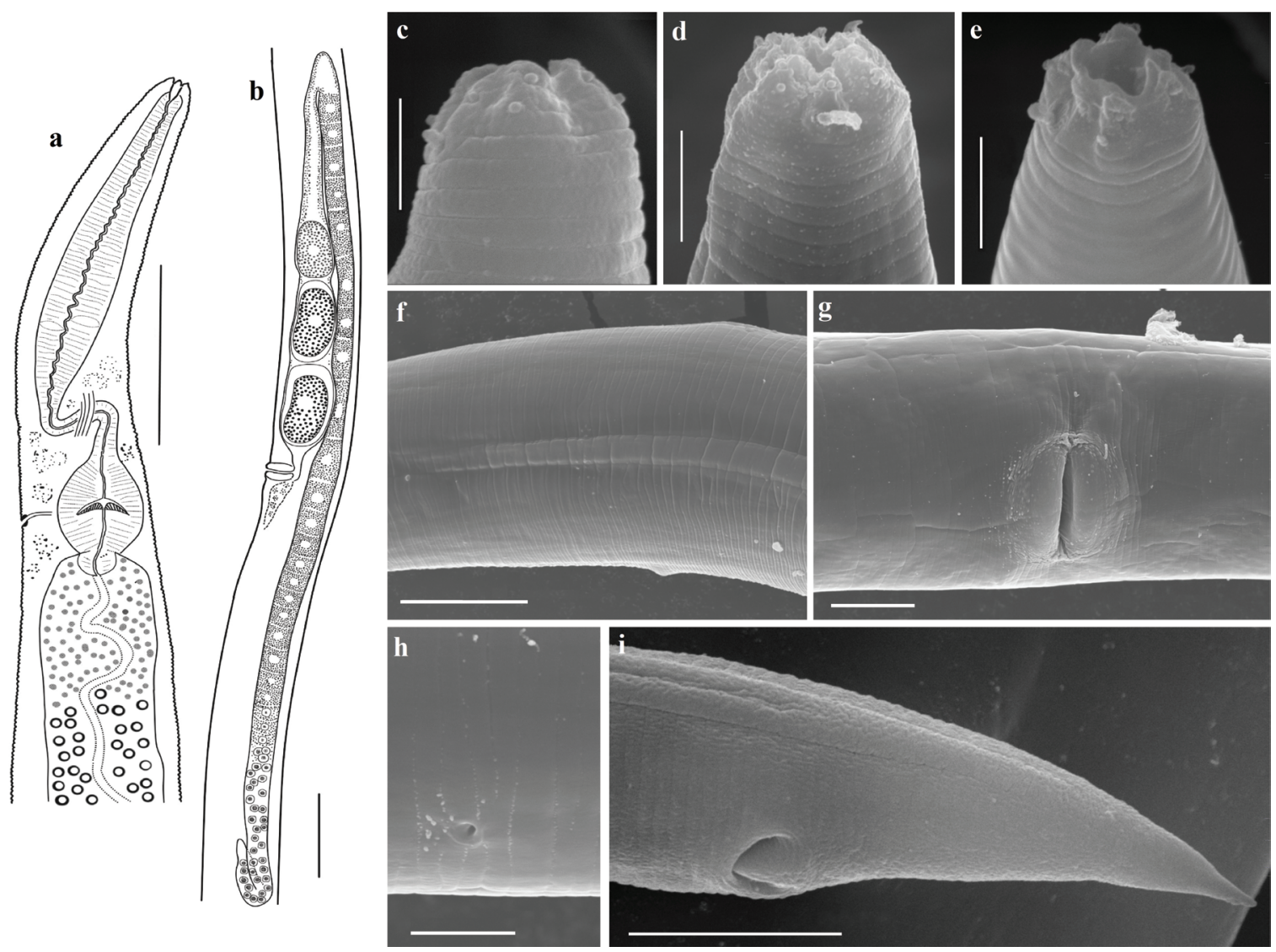

Figure S2

Shatilovich et al 
a

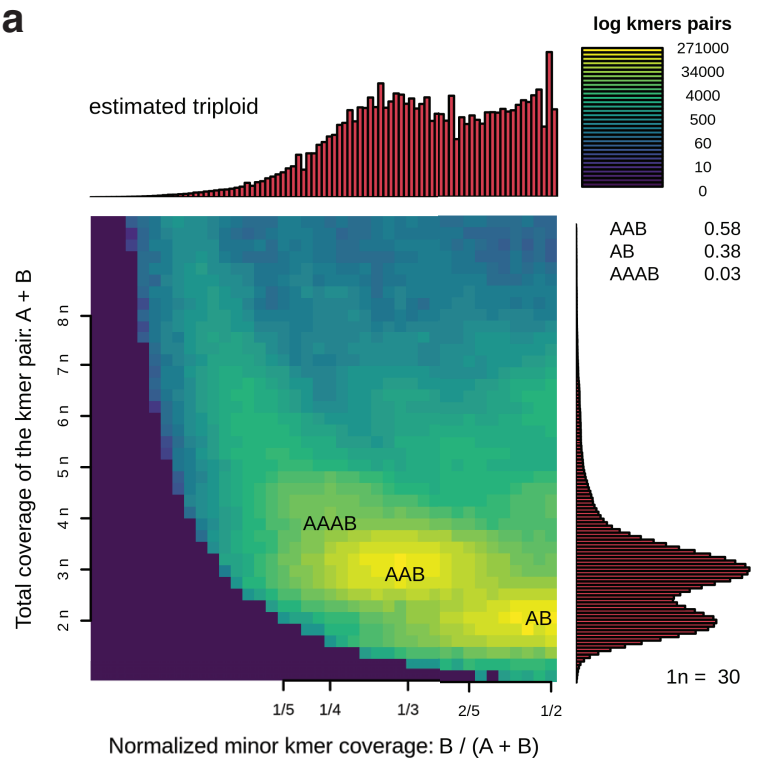

b

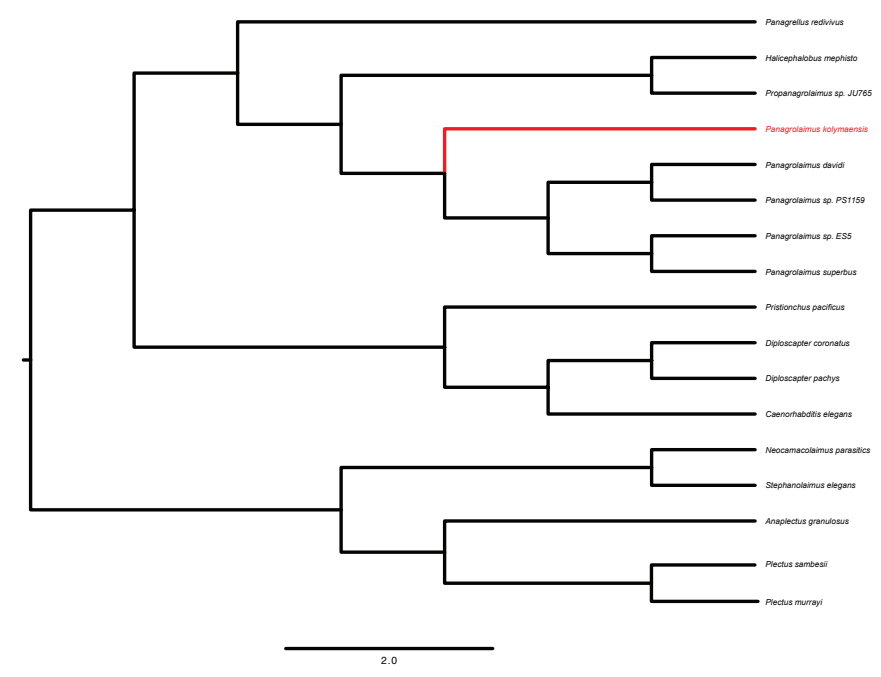

C

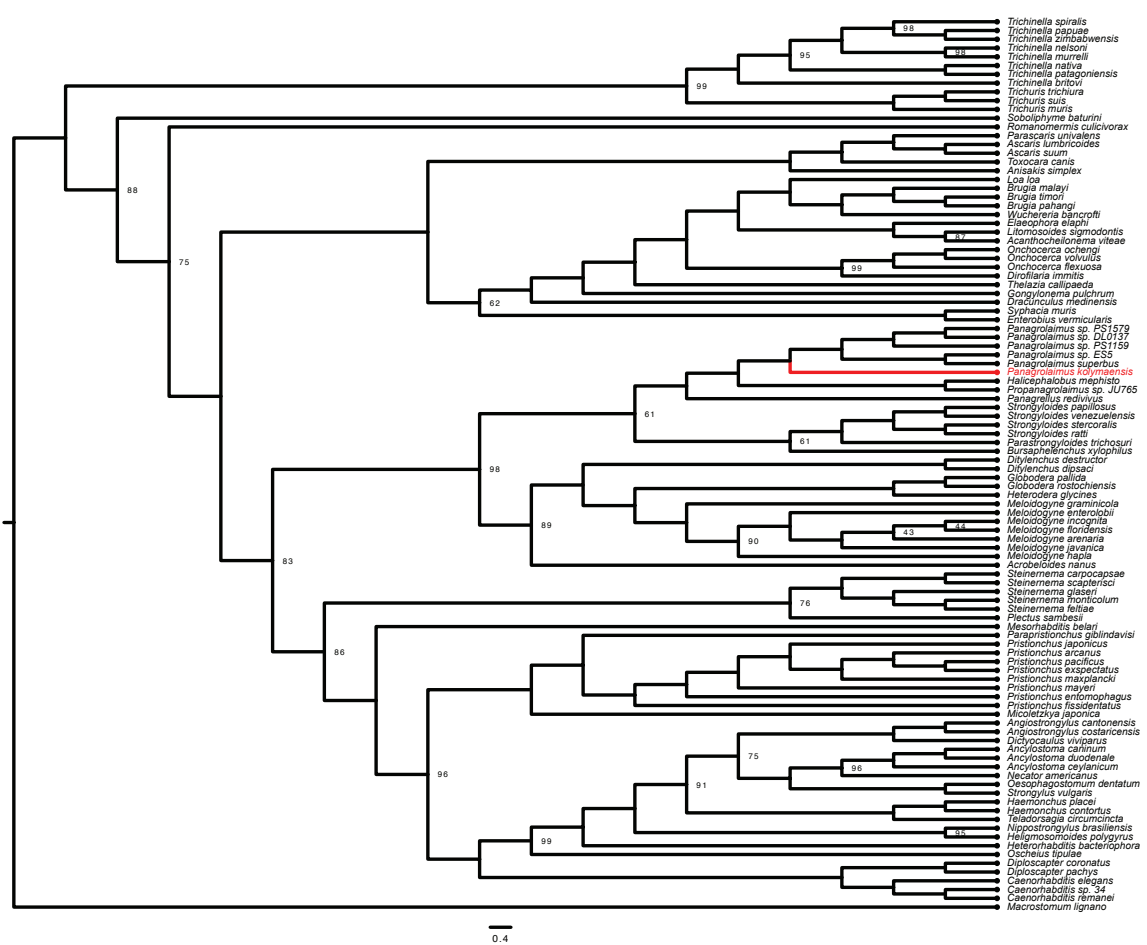

Figure S3

Shatilovich et al 
a

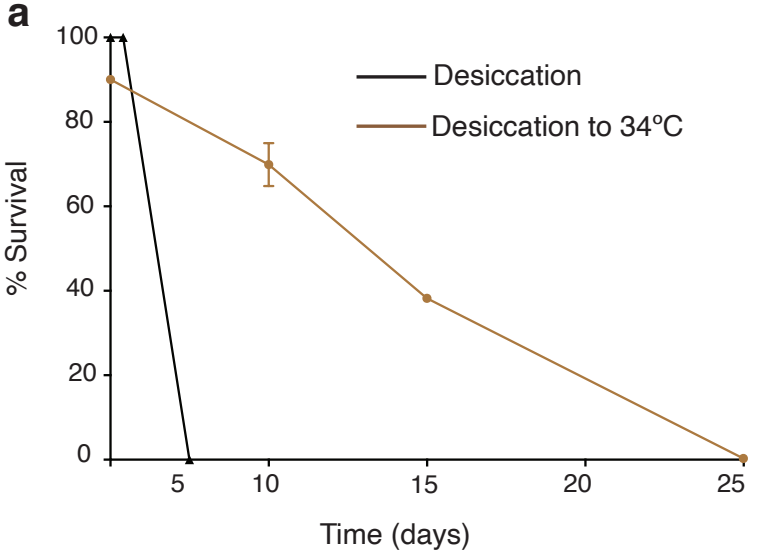

c

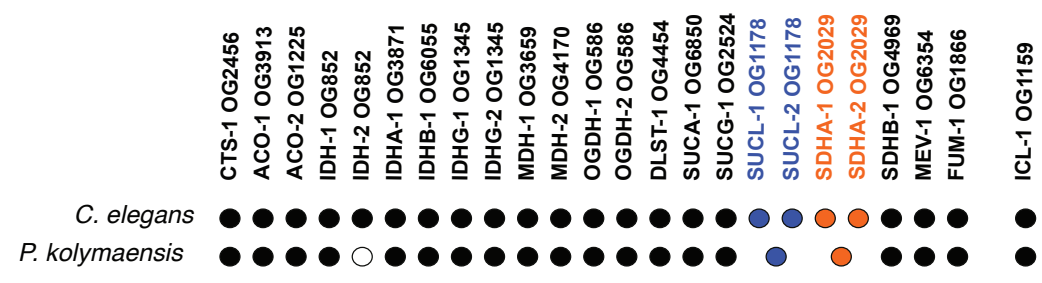

glycolysis/gluconeogenesis

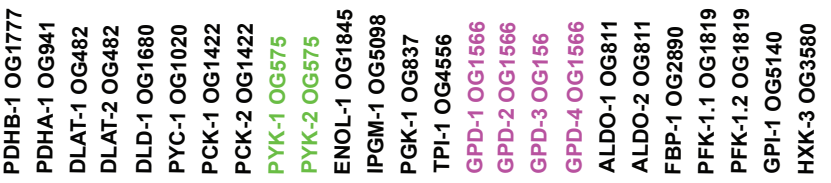

C. elegans

P. kolymaensis

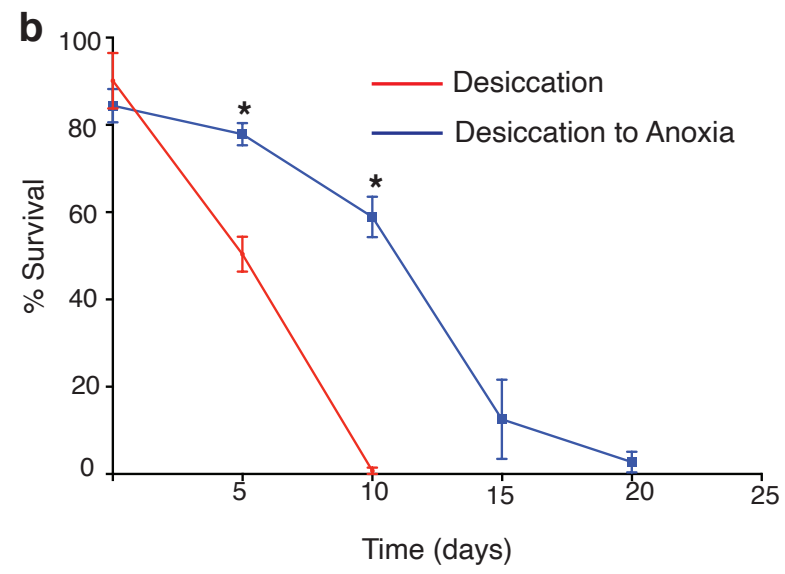

Trehalose synthesis

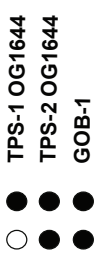

Polyamine synthesis

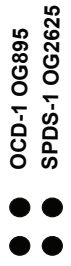

Figure S4

Shatilovich et al 
a

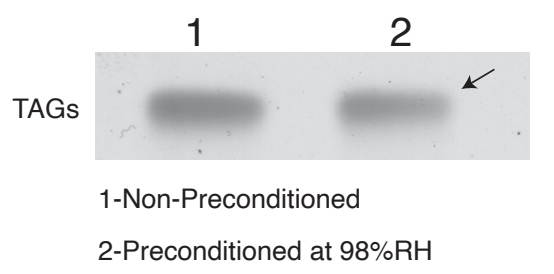

C

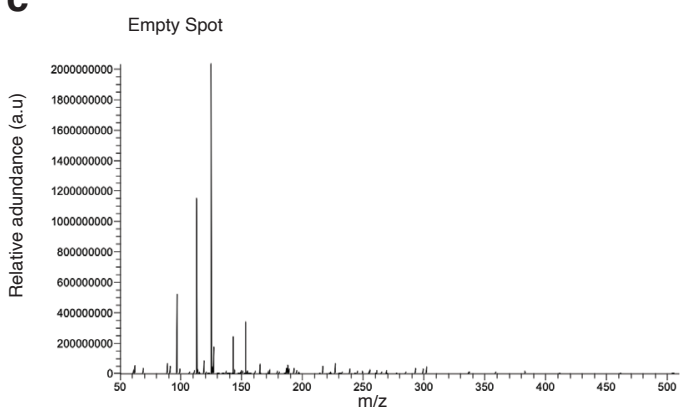

e

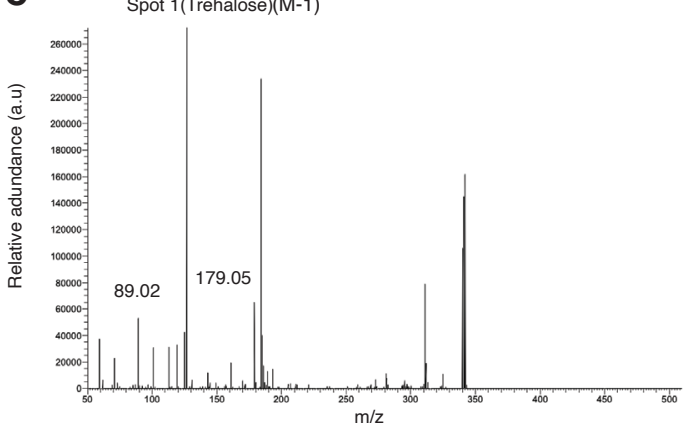

g

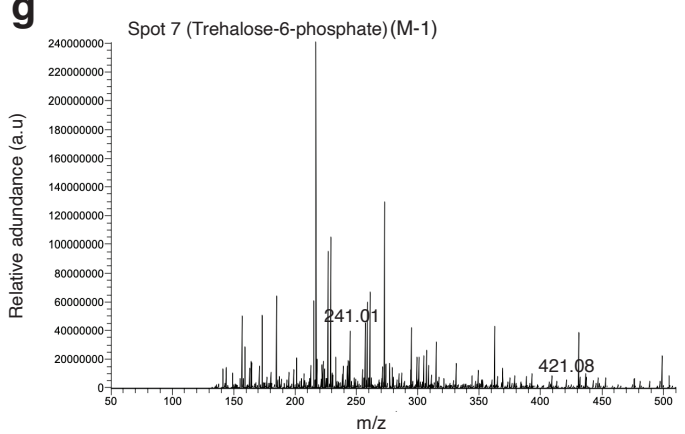

b
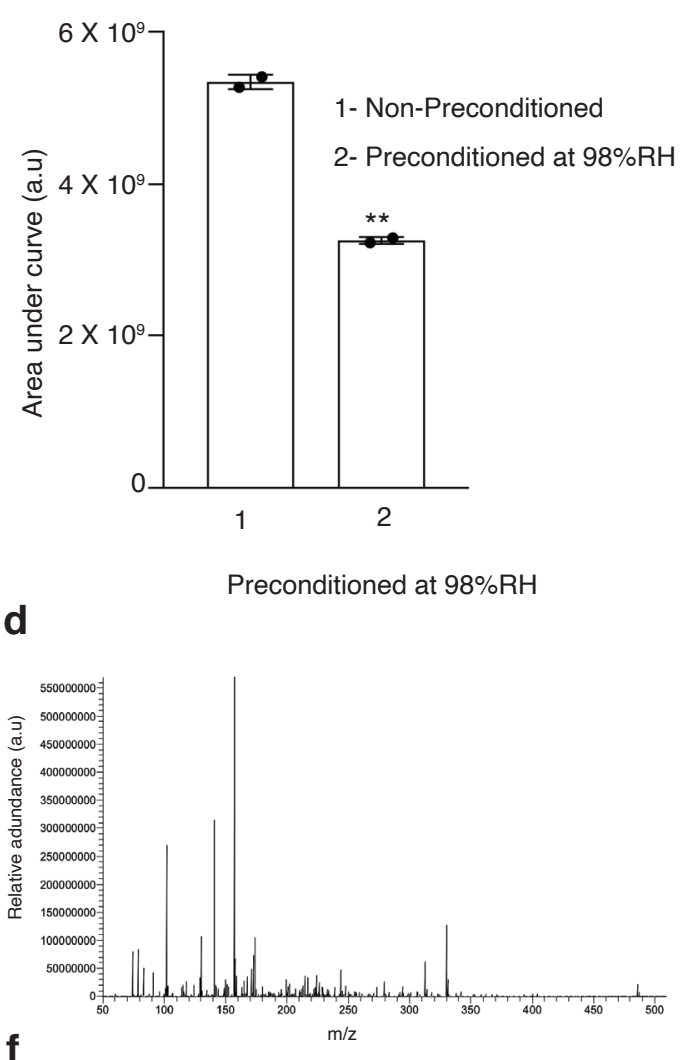

f

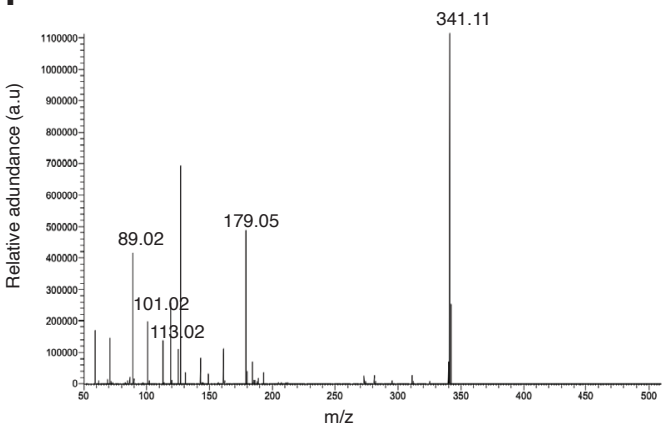

h

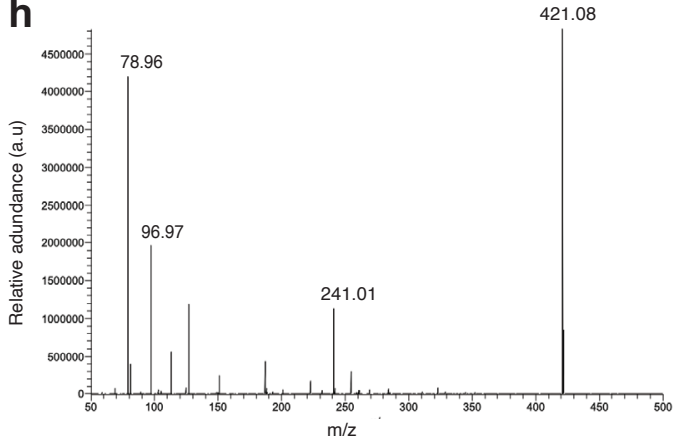

